\title{
Uréter circumcavo no obstructivo
}

\author{
E. Cao Avellaneda, G. Server Pastor, AI López López, A. Maluff Torres, P. López Cubillana, \\ M. Rigabert Montiel, M. Pérez Albacete
}

Servicio de Urología. Hospital "Virgen de la Arrixaca". Murcia.

Actas Urol Esp 2005; 29 (1): 107-109

\section{RESUMEN}

\section{URÉTER CIRCUMCAVO NO OBSTRUCTIVO}

Se presenta un caso de uréter circumcavo derecho no obstructivo o tipo dos de Batenson y Atkinson en un hombre de 49 años. Se diagnostico tras consulta por hematuria y dolor ureteral, atribuíble a infección urinaria concomitante. Destacamos la ausencia de retardo funcional, dilatación y morfología típica del mismo. Se decidió actitud expectante, estando asintomático tras 12 meses de seguimiento.

Palabras claves: Uréter retrocavo, uréter circumcavo, malformaciones.

\section{ABSTRACT \\ NON OBSTRUCTIVE RETROCAVAL URETER}

We report a case on circumcavum ureter without obstruction or type two, according the Batenson and Atkinson's classification, in a forty-nine years old man. It was diagnosticated for a gross haematuria and renoureteral pain, because of a simultaneous urinary tract infection. We emphasize its absence of the tipical morphology and obstruction signs. After twelve months the patient is still asintomatic, without any medical or surgical treatment.

Key Words: Retrocaval ureter, circumcavum ureter, malformations.

Se denomina uréter circumcavo a la anomalía del desarrollo venoso abdominal inferior donde el uréter rodea a la vena cava a nivel de tercera a cuarta vértebra lumbar. Se han descrito aproximadamente 400 casos en la literatura revisada. Batenson y Atkinson clasificaron esta alteración según la presencia o no de patrón obstructivo en dos tipos: el tipo 1, más frecuente, con hidronefrosis asociada, y tipo 2 en los casos con mínima o nula obstrucción. Su clínica suele ser obstructiva y tiene como prueba diagnóstica la TAC. El tratamiento es individualizado.

\section{CASO CLÍNICO}

Varón de 49 años que acude a consulta externa por hematuria terminal con dolor de tipo renoureteral izquierdo.

Entre sus antecedentes no cabe destacar ninguna alergia, enfermedad de afectación sistémica o intervención quirúrgica. Eneuresis nocturna primaria que se resolvió sin intervención médica a los 8 años de edad.

En la exploración física impresiona de varón sano. Normocoloreado y normohidratado. Cabeza, tórax, abdomen y miembros normal. Tacto rectal con próstata hipertrófica grado dos, indolora, lisa y bien delimitada. Resto de la exploración física sin hallazgos significativos.

Se realiza analítica de la sangre y orina a los diez días del episodio sin encontrar hallazgos patológicos. El valor de PSA es $1,2 \mathrm{ng} / \mathrm{ml}$. En la ecografía, la vejiga está libre de patología, sin defectos de replección. El urocultivo es positivo para Escherichia Coli. La citología urinaria es negativa. La cistoscopia sin hallazgos de interés. Se practica urografía intravenosa donde destaca la presencia de medialización de uréter derecho sin dilatación, litiasis o retardo de su función (Fig. 1), que obliga a la realización de TC con contraste, encontrando ambos riñones de tamaño y captación de contraste normal sin que existan imágenes que sugieran dilatación de los sistemas excretores. Se visualiza uréter circumcavo derecho sin signos de obstrucción o patología intraureteral (Figs. 2 y 3). Vía excretora izquierda normal. Resto del estudio dentro de la normalidad. 


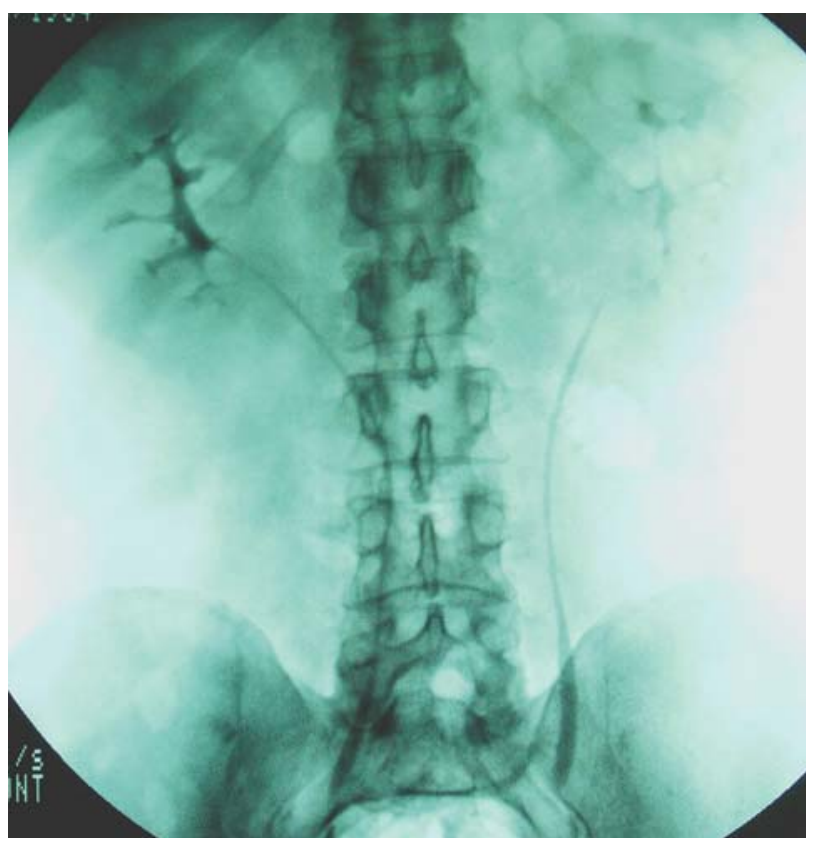

FIGURA 1. UIV. Se observa medialización del trayecto ureteral asociado a ausencia de signos obstructivos.

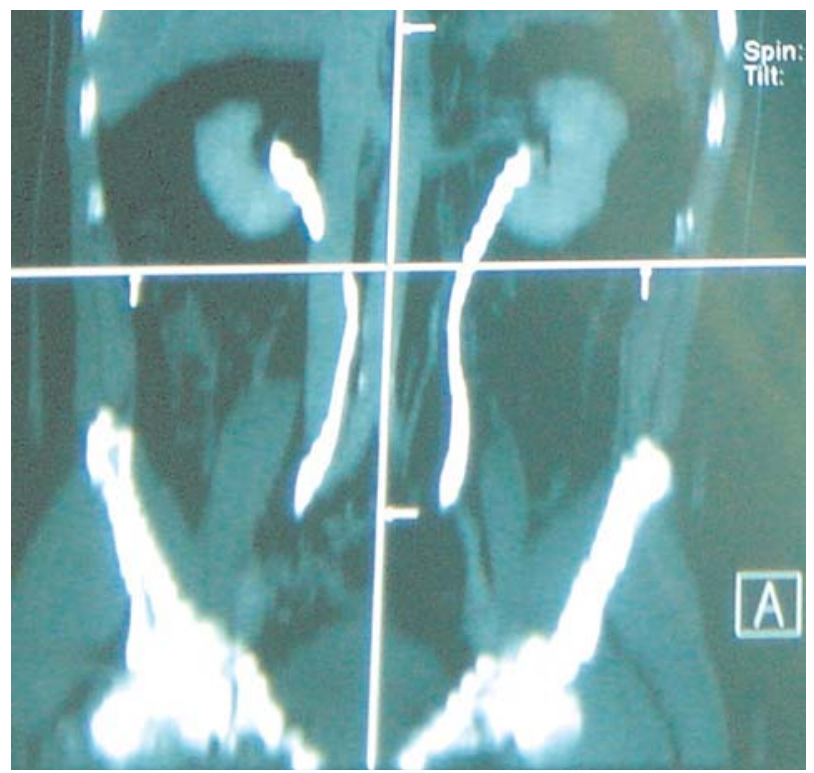

FIGURA 2. Imagen de TC mediante reconstrucción sagital.

Con el diagnóstico de uréter retrocavo no obstructivo o tipo 2, se decide actitud conservadora con revisión periódica, manteniendo igual estado tras 12 meses de seguimiento. Se trató la infección con beta lactámicos con éxito.

\section{DISCUSIÓN}

El término uréter retrocavo, describe esta anomalía anatómicamente, no así su génesis, por lo que algunos autores prefieren llamar a esta anor- malidad vena cava preureteral o uréter circumcavo, ya que es debido a una alteración del desarrollo venoso abdominal inferior y no del ureteral. Tres sistemas venosos están implicados en la formación de la vena cava inferior. La vena cardinal posterior que se origina en la cuarta semana como vasos longitudinales de localización dorsolateral, la vena subcardinal en la posición medial y la vena supracardinal. La persistencia de la vena subcardinal derecha es el origen de esta anormalidad (Fig. 4). Así, la alteración del trayecto ureteral sería secundario $^{1}$. Por estos motivos, preferimos denominarlo uréter circumcavo en nuestro artículo.

Fue descrita inicialmente por Hochstetter en 1873. Se han descrito aproximadamente 400 casos en la literatura desde entonces. Su incidencia se estima entorno a 1 de cada 1500 nacimientos, con predominio en hombres (4 a 1). Más frecuente en el uréter derecho, se asocia en el lado izquierdo a situs inversus ${ }^{2,3}$. En su forma más frecuente, cruza medial y posterior a la vena cava a nivel de la tercera o cuarta vértebra lumbar, dibujando el trayecto ureteral una forma en $\mathrm{S}$ o gancho. Tras esto, emerge entre cava y aorta y se sitúa nuevamente lateral para seguir su trayecto normal hasta vejiga. Esta alteración, como sucede en el caso que presentamos, puede no cursar con obstrucción. Batenson y Atkinson clasificaron esta alteración según la presencia o no de patrón obstructivo en dos tipos: el tipo 1, más frecuente, con hidronefrosis asociada, y tipo 2 en los casos con mínima o nula obstrucción ${ }^{4}$. Malformación congénita , se ha asociado al contacto de las madres con éter dietileno monometil ${ }^{5}$. Hasta un $20 \%$ de los pacientes asocian otras malformaciones, generalmente renales, vasculares y genitales ${ }^{6,7}$.

La clínica típica de inicio en el tipo 1 suele relacionarse con la ectasia de causa mecánica o funcional (este segmento retrocavo puede ser hipoplásico $^{8}$. Con todo, no es raro que se descubra en la tercera o cuarta década de la vida. El diagnóstico suele iniciarse tras la realización de una urografia intravenosa, donde se visualiza la típica medialización ureteral con segmento superior en gancho o $\mathrm{S}$ y la porción retrocava a nivel de $\mathrm{L}_{3}-\mathrm{L}_{4}$. La TC es diagnóstica (Figs. 2 y 3). La ecografía y el renograma isotópico aportan información sobre el patrón obstructivo o no del mismo, clasificándolo en tipo 1 ó 2. El diagnóstico diferencial incluye masas en retroperitoneo y fibrosis retroperitoneal. 


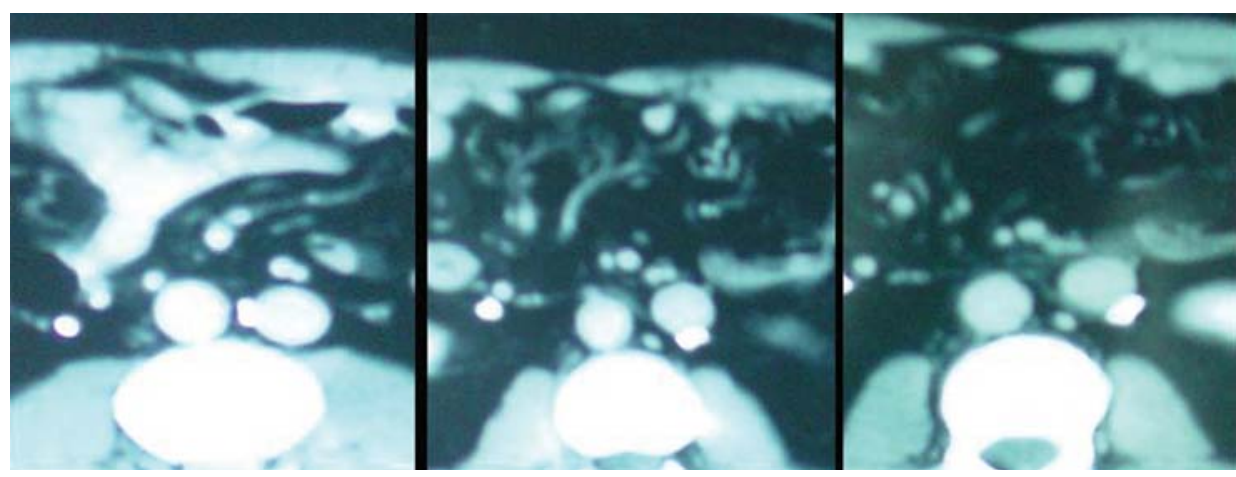

FIGURA 3. Detalle TC; se observa recorrido de uréter a nivel de la vena cava.
En conclusión, el uréter retrocavo es una rara alteración, fácilmente diagnosticable y con posibilidad de tratamiento eficaz.

Debe evaluarse la posibilidad de otras malformaciones asociadas que puedan modificar la actitud terapéutica.

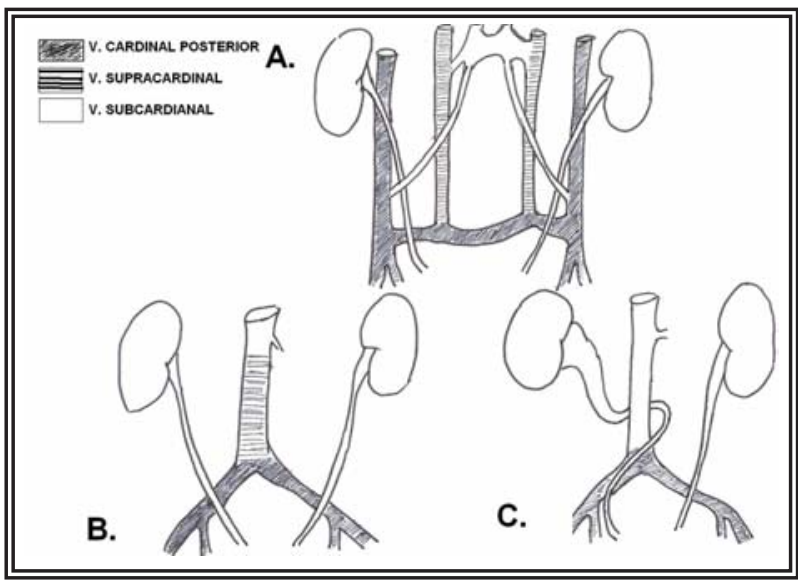

FIGURA 4. Esquema de la embriología del uréter circumcavo.

Si es necesaria la corrección quirúrgica, ésta requiere un abordaje individualizado dependiendo del grado de alteración y malformaciones asociadas. Clásicamente se ha descrito la escisión y abandono del segmento retrocavo, generalmente adinámico, y la reanastomosis término-terminal. El principio que debe regir cualquier maniobra es la conservación del aporte vascular del trayecto craneal (dependiente de aorta) y caudal al mismo (dependiente de iliaca). Sólo en el caso de riñón único se podría plantear la inversión del proceso, con división y recolocación de la vena cava. El primer caso de resolución mediante abordaje laparoscópico fue comunicado a principios de 1996 por Matsuda ${ }^{9}$, que sigue los mismos principios indicados, habiéndose publicado hasta la actualidad múltiples casos tratados por esta vía ${ }^{10-13}$.

La singularidad de este caso radica en la presentación clínica tardía y fundamentalmente en la ausencia de patrón obstructivo, poco comunicado en la literatura revisada.

\section{BIBLIOGRAFÍA}

1. Gay SB, Armistead JP, Weber ME et al. Left infrarrenal region: anatomic variants, pathologic conditions, and diagnostic pitfalls. Radigraphics. 1991;11:550.

2. Pierro JA, Soleimanpour M, Bory JL. Left retrocaval ureter associated with left inferior vena cava. AJR.1990; 155:545.

3. Rubinstein I, Calvacanti AG, Canalini AF, Freitas MA, Accioly PM. Left retrocaval ureter associated with inferior vena caval duplication. J Urol. 1999; 162: 1373-4.

4. Bateson EM. Atkinson D.Circuncaval ureter: a new classification. Clin Radiol.1969;20:173.

5. Karaman MI, Gurdal M, Ozturz M. Maternal exposure to diethylene glycol monomethyl ether: a possible role in the etiology of retrocaval ureter. J Pediatr Surg. 2002; 37(8): E23.

6. Perimenis P, Gyftopoulos K, Athanasopoulos et al. Retrocaval ureter and associated abnormalities. Int Urol Nephrol. 2002;33:19-22.

7. Ritchey ML, Norbeck J, Huang C, Keating MA y Bloom. Urologic manifestations of Goldenhar syndrome. Urology. 1994; 88:43.

8. Soundappan SV, Barker AP. Retrocaval ureter in children: a report of two cases. Pediatr Surg Int. 2004; 20(2): 158-60.

9. Matsuda T, Yasumoto and Tsujino T. Laparoscopic treatment of retrocaval ureter. Eur Urol.1996; 29(115): 115.

10. Polanscik TJ, Chen RN. Laparoscopic ureteroureterostomy for retrocaval ureter. J Urol.1998; 160(1): 121-2.

11. Ameda K, Kakizaki H, Harabayashi K. Laparoscopic ureteroureterostomy for retrocaval ureter. Int J Urol.2001; 8(2):71-4.

12. Miyazato M, Kimura T, Ohyama C, Hatano T, Miyazato T, Ogawa Y. Retroperitoneoscopic ureteroureterostomy for retrocaval ureter. Hinyokika Kiyo.2002; 48(1): 25-8.

13. Bhandarkar DS, Lalmalani JG, Shive S. Laparoscopic ureterolysis and reconstruction of a retrocaval ureter. Surg Endosc. 2003; 17(11): 1851-2.

14. Kogan BA. Transtornos del uréter y la unión ureteropiélica. En Urología General de Smith. Tanagho EA, Mc Aninch JW. $12^{\circ}$ edición en español. Editorial El Manual Moderno. México DF. 2001.

15. Resel Estévez L. Ureter retro-cava. En: Clínicas Urológicas de la Complutense. Patología vascular en Urología. Resel L y Salinas J.Editorial Complutense. Madrid. 1992.

Dr. Enrique Cao Avellaneda

C/ Mateos, no $19^{\circ}$ C.

30002 Murcia

e-mail: enriquecao@hotmail.com

(Trabajo recibido el 31 mayo 2004) 
